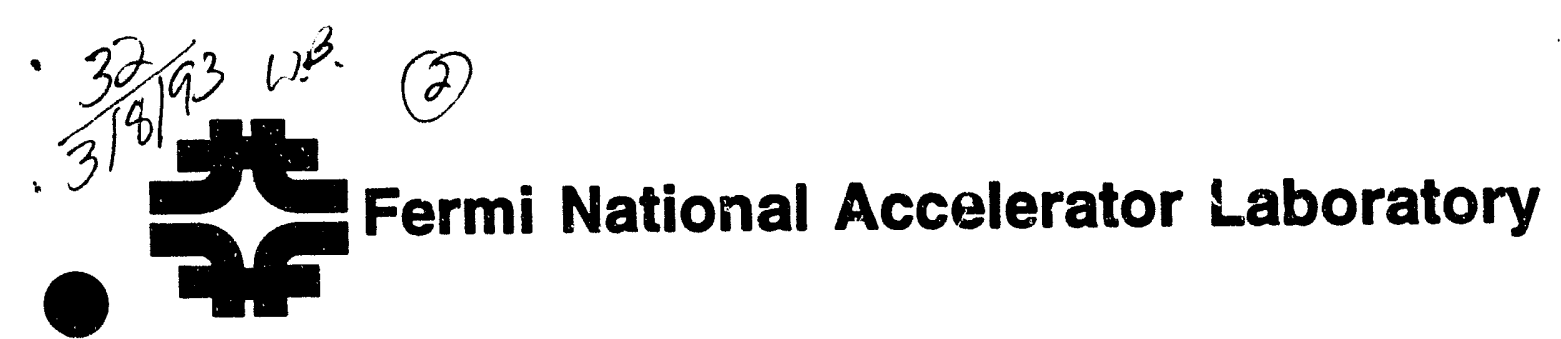

FNAL-TM--1826

DE93 008371

\title{
Magnetic Field Effects on Endcap EM Calorimetry in SDC
}

\author{
Dan Green \\ Fermi National Accelerator Laboratory \\ P.O. Box 500, Batavia, Illinois 60510
}

\section{DISCLAIMER}

This report was prepared as an account of work sponsored by an agency of the United States Government. Neither the United States Government nor any agency thereof, nor any of their employees, makes any warranty, express or implied, or assumes any legal liability or responsibility for the accuracy, completeness, or usefulness of any information, apparatus, product, or process disclosed, or represents that its use would not infringe privately owned rights. Reference herein to any specific commercial product, process, or service by trade name, trademark, manufacturer, or otherwise does not necessarily constitute or imply its endorsement, recommendation, or favoring by the United States Government or any agency thereof. The views and opinions of authors expressed herein do not necessarily state or reflect those of the United States Government or any agency thereof.

January 1993

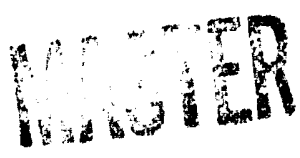




\section{Disclaimer}

This report was prepared as an account of work sponsored by an agency of the United States Government. Neither the United States Government nor any agency thereof, nor any of their employees, makes any warranty, express or implied, or assumes any legal liability or responsibility for the accuracy, completeness, or usefulness of any information, apparatus, product, or process disclosed, or represents that its use would not in, iringe privately owned rights. Reference herein to any specific commercial product, process, or service by trade name, trademark, manufacturer, or otherwise, does not necessarily constitute or imply its endorsement, recommendation, or favoring by the United States Government or any agency thereof. The views and opinions of authors expressed herein do not necessarily state or reflect those of the United States Government or any agency thereof. 


\title{
MAGNETIC FIELD EFFECTS ON ENDCAP EM CALORIMETRY IN SDC
}

\author{
Dan Green \\ Fermilab \\ Batavia, Illinois \\ January 25, 1993
}

\section{Introduction}

The SDC calorimeter will be immersed in a strong magnetic field in the endcap region because of the solenoid which supplies the SDC tracking field. This flux must be returned through the endcap region of the electromagnetic (EM) and hadronic (HAD) calorimeters. Since magnetic fields are known to induce changes in the light output of plastic scintillator, the endcap will need to be recalibrated once the solenoid is energized. In addition, gradients in the field will create nonuniformities in the calorimetric response. The "induced constant term" in the EM and HAD calorimeters due to the inhomogeneities induced by the magnetic field are here evaluated in order to see that SDC performance specifications are not compromised.

\section{The Magnetic Field and Plastic Scintillator}

The magnetic field in SDC has been modeled [1] in 3 dimensions. The mesh for this model is sufficiently fine that gradients are easily evaluated. In Fig. 1 is shown the total field strength, in Tesla, as a function of longitudinal location (z) in the EM endcap. The radii shown span the region occupied by the endcap calorimeters. Typical longitudinal gradients are $2 \mathrm{kG}$ across the EM endcap at both small and large radii. In comparison, the field strength in the HAD1 compartment $(5.0<z<5.9 \mathrm{~m})$ is shown in Fig. 2 as a function of $\mathrm{z}$ for several radii. The gradient is $\sim 14 \mathrm{kG}$ across the longitudinal extent of HAD1 in this case. Although the gradients are large, the SDC specification is sufficiently loose that no new provisions in hadronic calorimetry are required [2].

The fractiond shift [3] in light output for SCSN38 scintillator is shown in Fig. 3. A linear representation leads to a slope of $0.6 \% / \mathrm{kG}$. Looking at Fig. 2, that slope means that the HAD1 compartment in the endcap sees a light output gradient of $<6.4 \%$ with a shift (to be recalibrated) of a few \% [2]. For the EM region, a roughly uniform field of $12 \mathrm{kG}$ leads to a shift of $\sim 8 \%$, while a gradient of $2 \mathrm{kG}$ causes a longitudinal inhomogeneity of $1.2 \%$.

The transverse segmentation of the SDC EM endcap is shown in Fig. 4. The basic tile size in $(\eta, \phi)$ space is $(0.05,0.05)$. At large $\eta$, the EM shower would become a large fraction of this tile size, and consequently, the segmentation is increased [4]. In the region of $\eta$ from 2 to 3 , the field has a transverse gradient of $\sim 5 \mathrm{kG} / \mathrm{m}$. Converting from radius to $\eta$ 
using Fig. 4, one finds that over an EM tower, the field gradient is $\sim 0.6 \mathrm{kG}$. At lower $\eta$, from 1.4 to 2 in $\eta$, the field gradient is also roughly $5 \mathrm{kG} / \mathrm{m}$. A unit of $\eta$ is physically larger, but the segmentation of a tower is finer. Consequently, the gradient over an EM tower is again $\sim 0.4$ to $0.6 \mathrm{kG} /$ tower. Therefore, the transverse gradients in the SDC EM endcap calorimeter lead to $\sim 0.36 \%$ nonuniformities in light output. Since the allowed transverse nonuniformity in a tile has already been evaluated to be $\leq 2 \%$ [5], one can conclude that transverse gradients are under control. It remains to evaluate the effect of the longitudinal gradients on the energy response of the EM calorimeter.

\section{EM Showers and Longitudinal Gradients}

Rather than using the EGS Monte Carlo, test beam data from the "Hanging File" (HF) test calorimeter was used. The EM compartment in one incarnation of the HF stack consisted of 40 layers of $1 / 8 " \mathrm{~Pb}$, which is quite similar to the SDC configuration [5]. That configuration data set [6] was used for this analysis. Typical event profiles of $170 \mathrm{GeV}$ electron showers are shown in Fig. 5. Layer numbers 41 to 95 correspond to 45 plates of 1 "Fe. Note the quite uniform shower development of the electron showers. Fluctuations are fairly small, and are expected to consist largely of a shift of the shower shape by plus and minus about 1 radiation length. This shift consists in the smearing of a fixed shower shape by the fluctuations in the conversion point.

A model calorimeter with a $40 \%$ longitudinal gradient was "constructed" and HF electron data were thrown upon it. The resulting fractional energy shift had a mean of $24 \%$. The distribution of this shift is shown in Fig. 6a. The rms of that distribution is $1.62 \%$. Note that the homogeneous input HF data had a $1.5 \% \mathrm{~ms}$ at this energy. Therefore, the $40 \%$ gradient induced an uncorrected $0.61 \%$ constant term into the energy resolution. Note that the expected gradient throughout the EM compartment is expected to be $<6.4 \%$. Therefore, since the error is roughly linear in the magnitude of the gradient, the real effect is expected to be well within the SDC specifications [5].

Furthermore, the gradient causes a longitudinal inhomogeneity. Longitudinal segmentation can be used to reduce the effect of this type of inhomogeneity, as is the case for radiation induced nonuniformity. In Fig. $6 \mathrm{~b}$ is shown the correlation between the energy at plate 9 , the "shower maximum" (SM) detector and the fractional energy shift for a $40 \%$ gradient. Clearly, the effect of that gradient can be reduced by using SM information, just as it is used in reducing the induced constant term due to radiation damage. 


\section{References}

1. B. Wands private communication.

2. D. Green, "Magnetic Fields and SDC Endcap Scintillator Performance", FermilabTM-1923, SDC-93-404, December 1992.

3. J. Mainusch et al., Nucl. Inst. Meth. A312 451 (1992).

4. SDC Parameters Book, SDT-000010, September 14, 1992.

5. SDC Technical Design Report, SDC-92-101 (1992).

6. A. Byon private communication.

7. A. Beretvas et al., "Beam Tests of Composite Calorimeter Configurations from Reconfigurable-Stack Calorimeter", accepted by Nuc. Inst. Meth. 

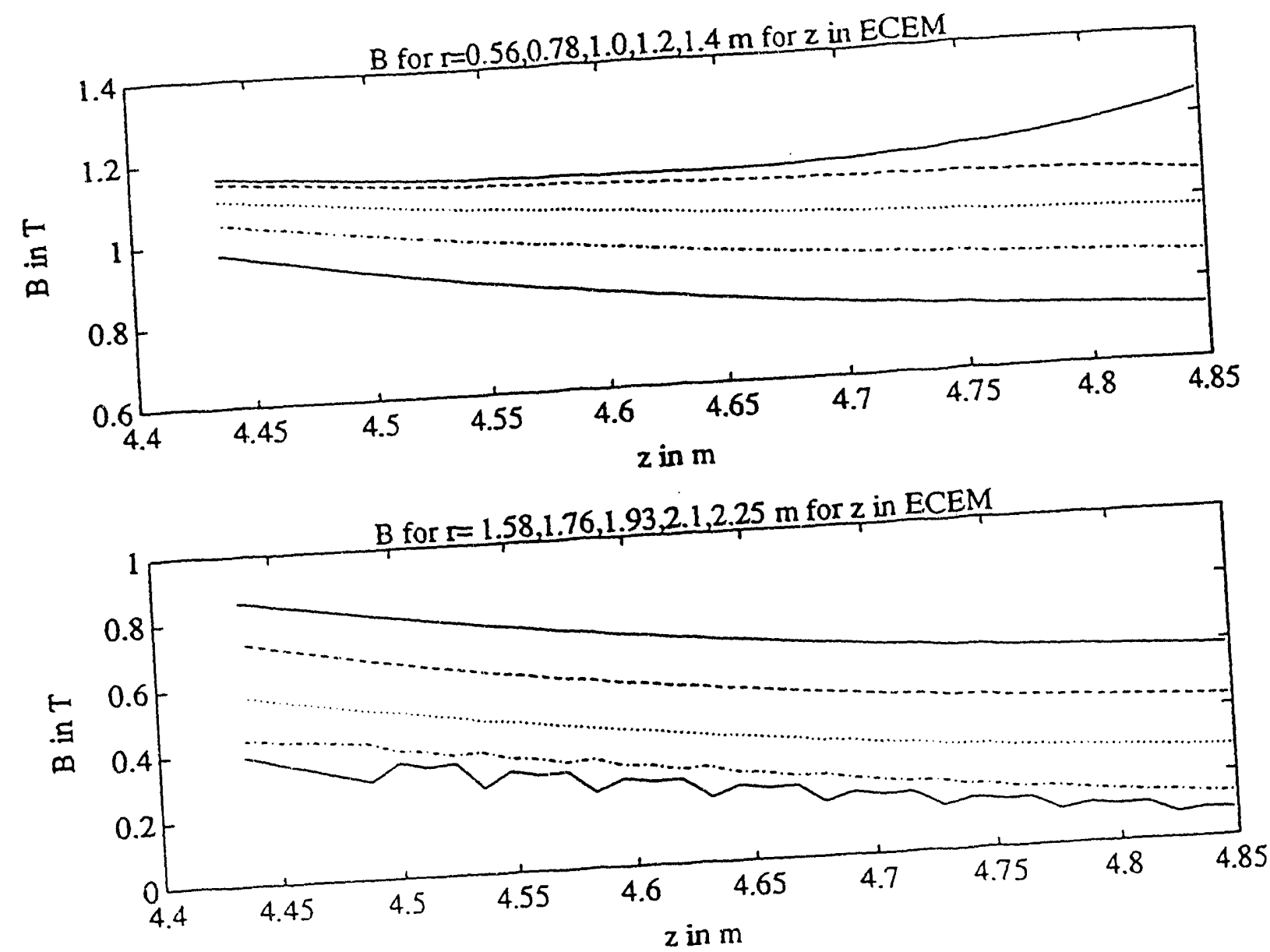

Fig. 1 Magnetic field strength as a function of depth in the SDC EM calorimeter in the endcap region.

a. radius $=0.56,0.78,1.0,1.2$ and $1.4 \mathrm{~m}$

b. radius $=1.58,1.76,1.93,2.1$ and $2.25 \mathrm{~m}$ 


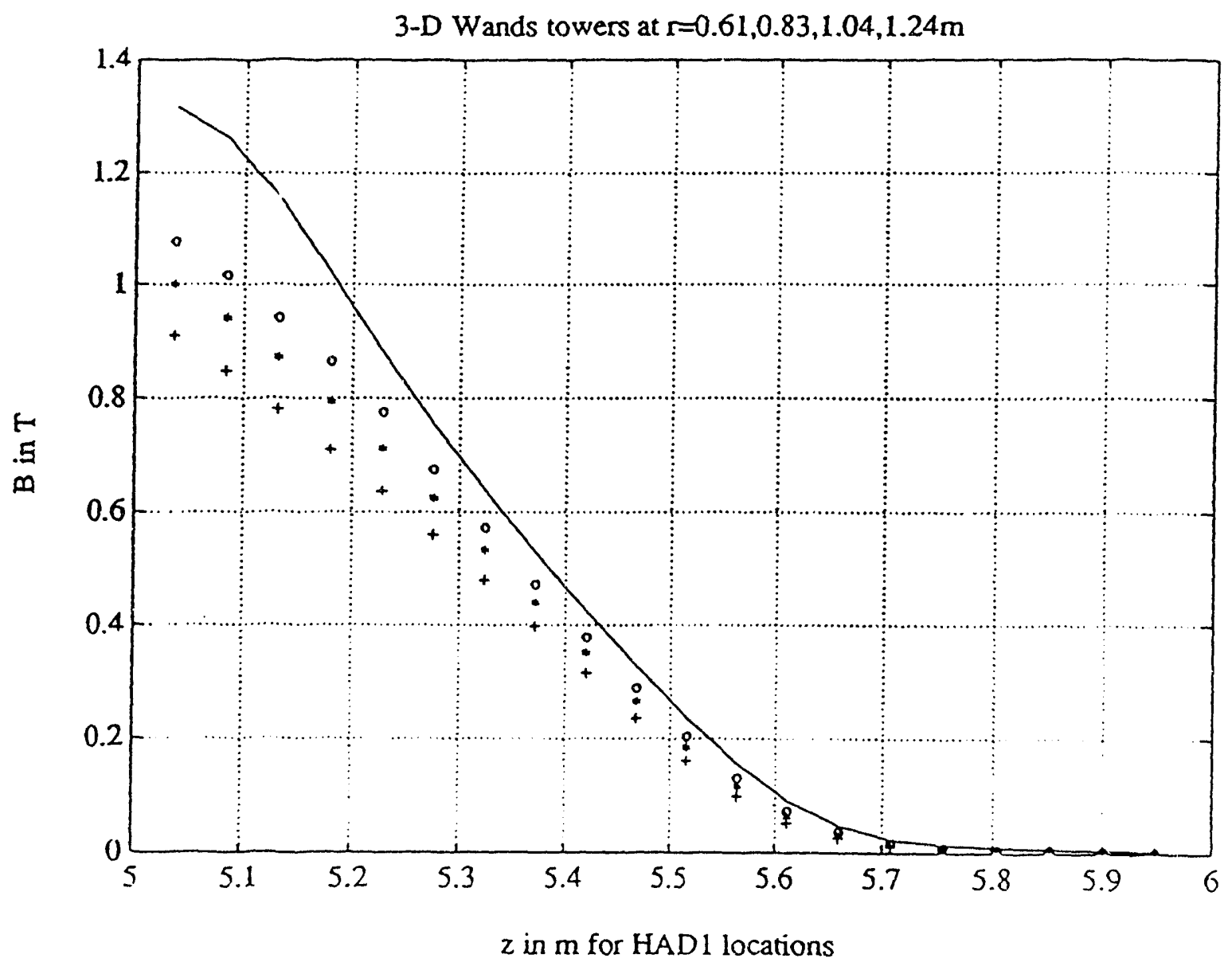

Fig. 2 Magnetic field strength as a function of depth in the SDC HAD1 calorimeter in the endcap region. The radii are $=0.61,0.83,1.04$ and $1.24 \mathrm{~m}$. 


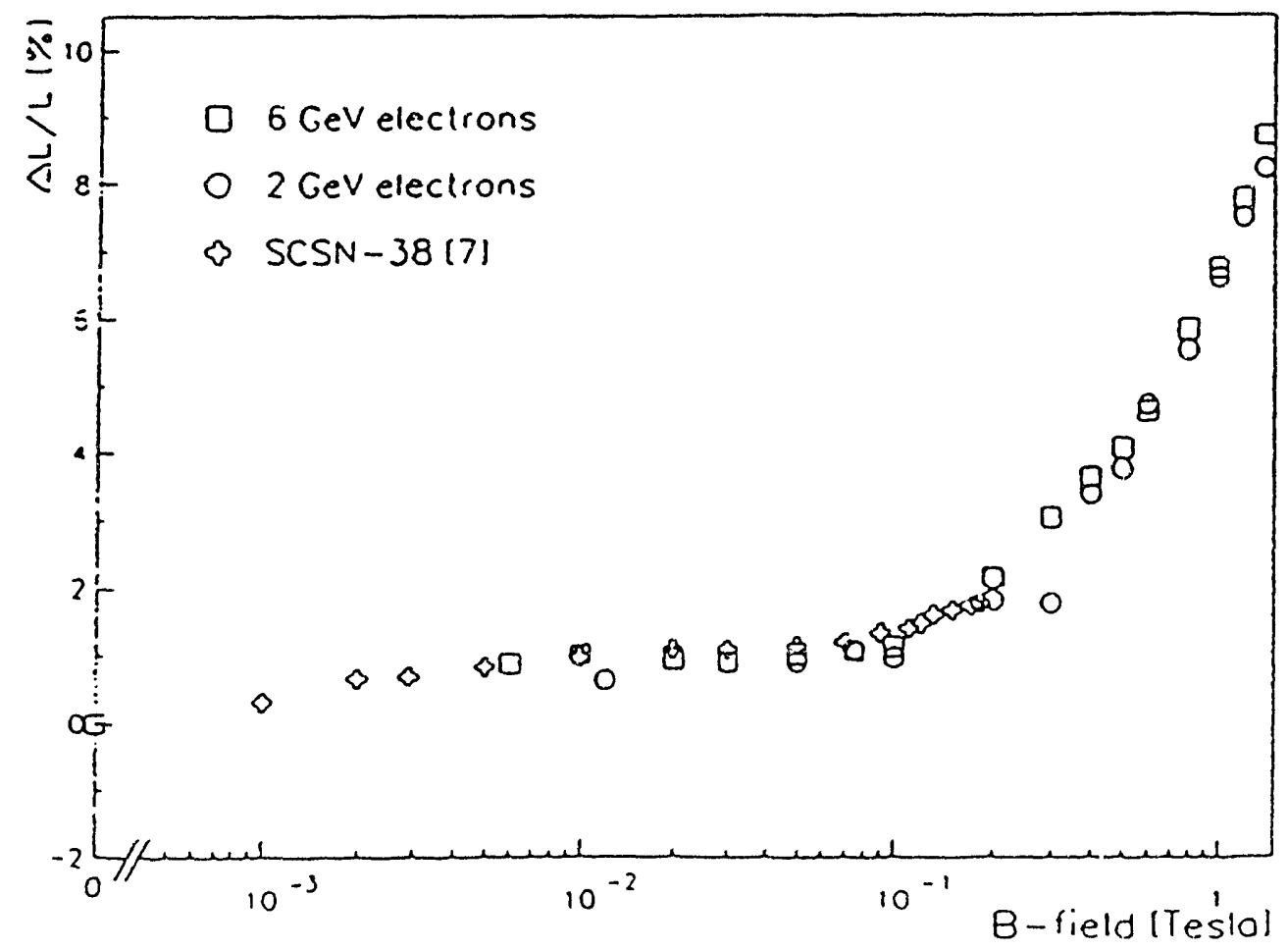

Fig. 3 Fractional light output change in SCSC38 plastic scintillator as a function of applied magnetic field strength. A linear relationship has a slope of roughly $0.6 \% / \mathrm{kG}$. 


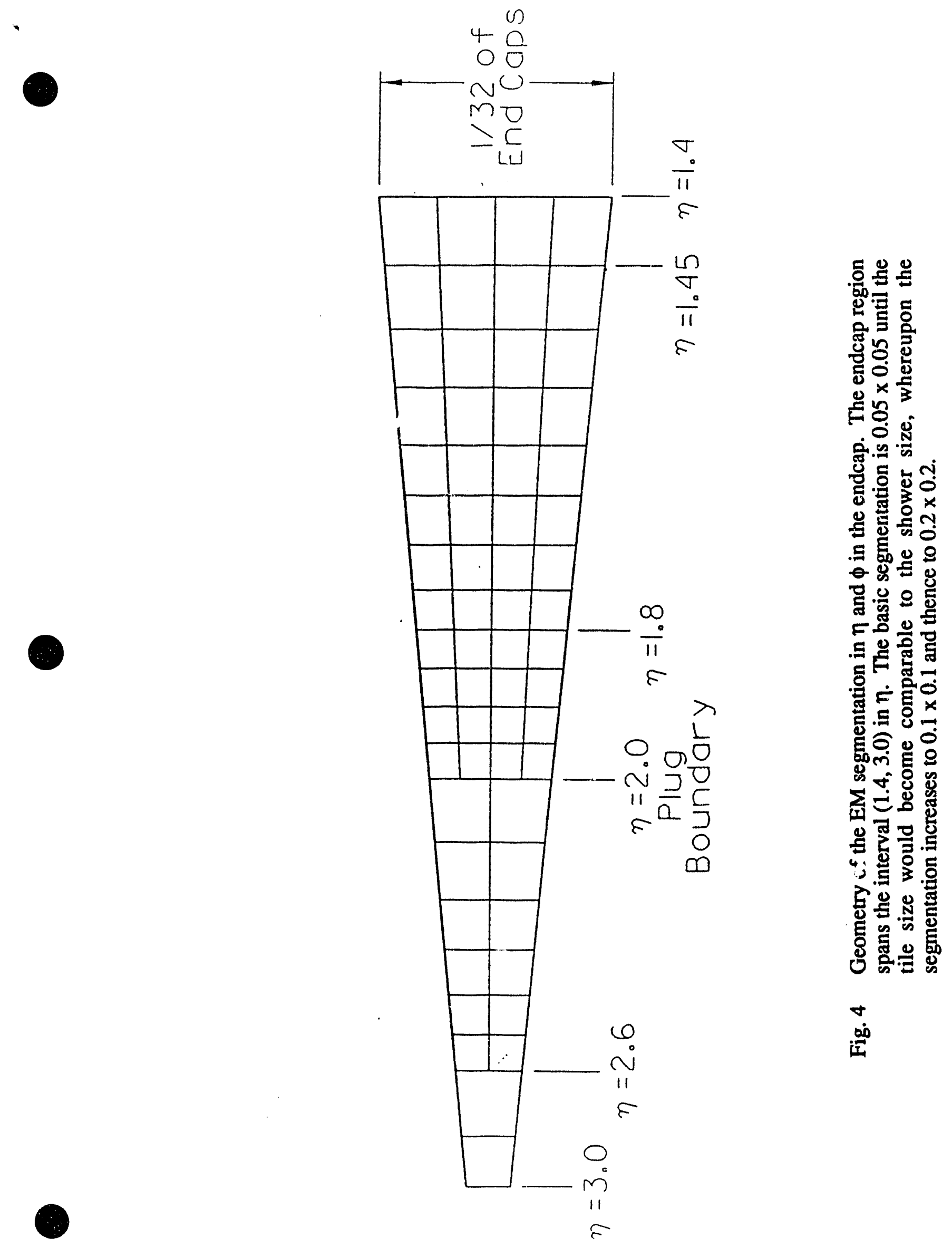



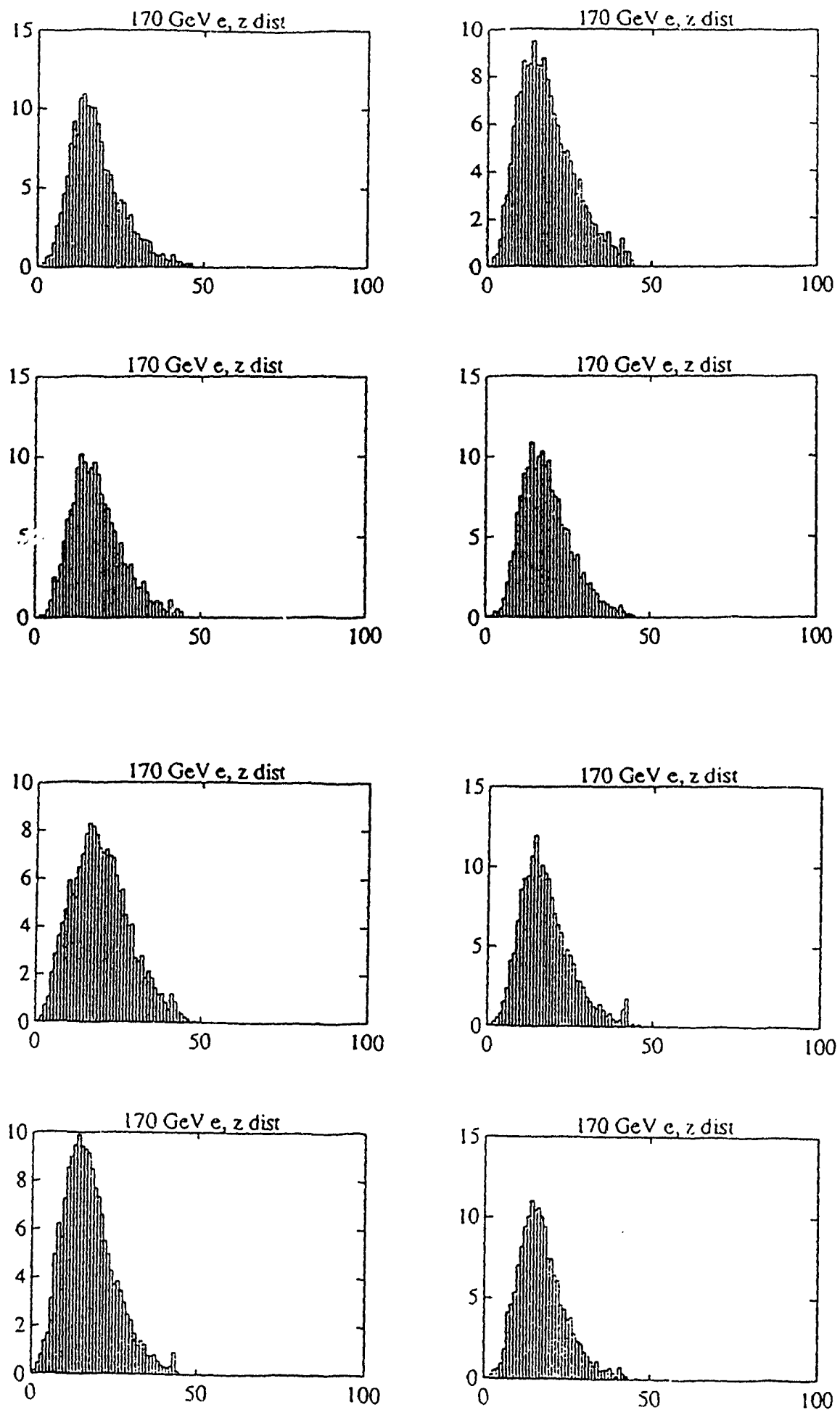

Fig. 5 Longitudinal shower distributions of 8 typical electron showers. The data is from the HF test beam set, and the stack consists of 40 plates of $1 / 8^{\prime \prime} \mathrm{Pb}$ initially, followed by 55 plates of 1 " Fe. Note the uniformity in shape of the electron showers. The mean energy for many showers is $171 \mathrm{GeV}$ with a rms/mean of
$1.5 \%$. 

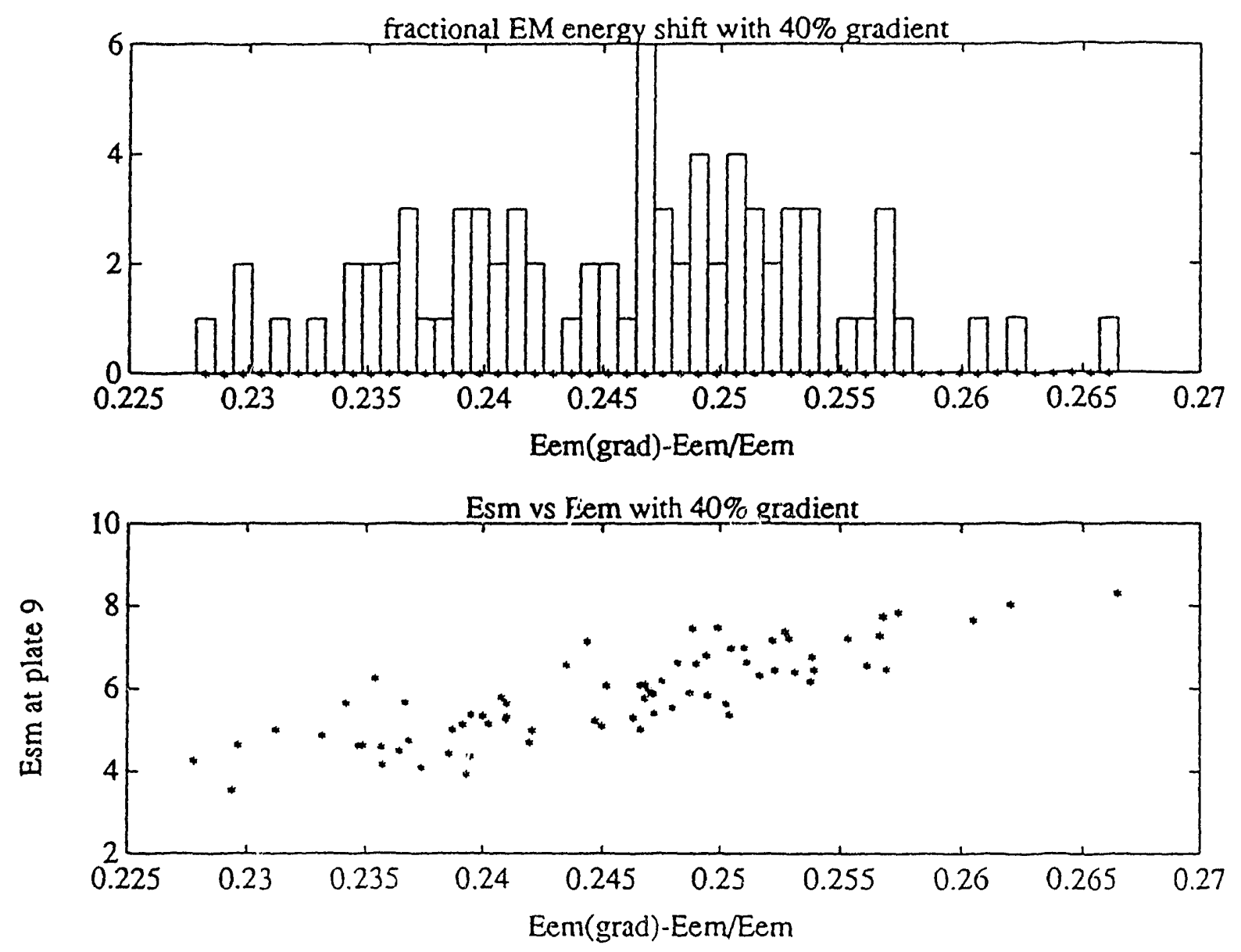

Fig. 6 a. Fractional energy shift for a $40 \%$ longitudinal gradient in the EM light output response. The mean shift is $24.5 \%$ with a $\mathrm{ms}=1.62 \%$. This implies an
induced constant term of $0.61 \%$.

b. Energy deposited at tile \# 9 (SM) plotted against the fractional energy shift for a $40 \%$ longitudinal EM gradient. The observed correlation means that Esm information may be used to reduce the induced constant term. 

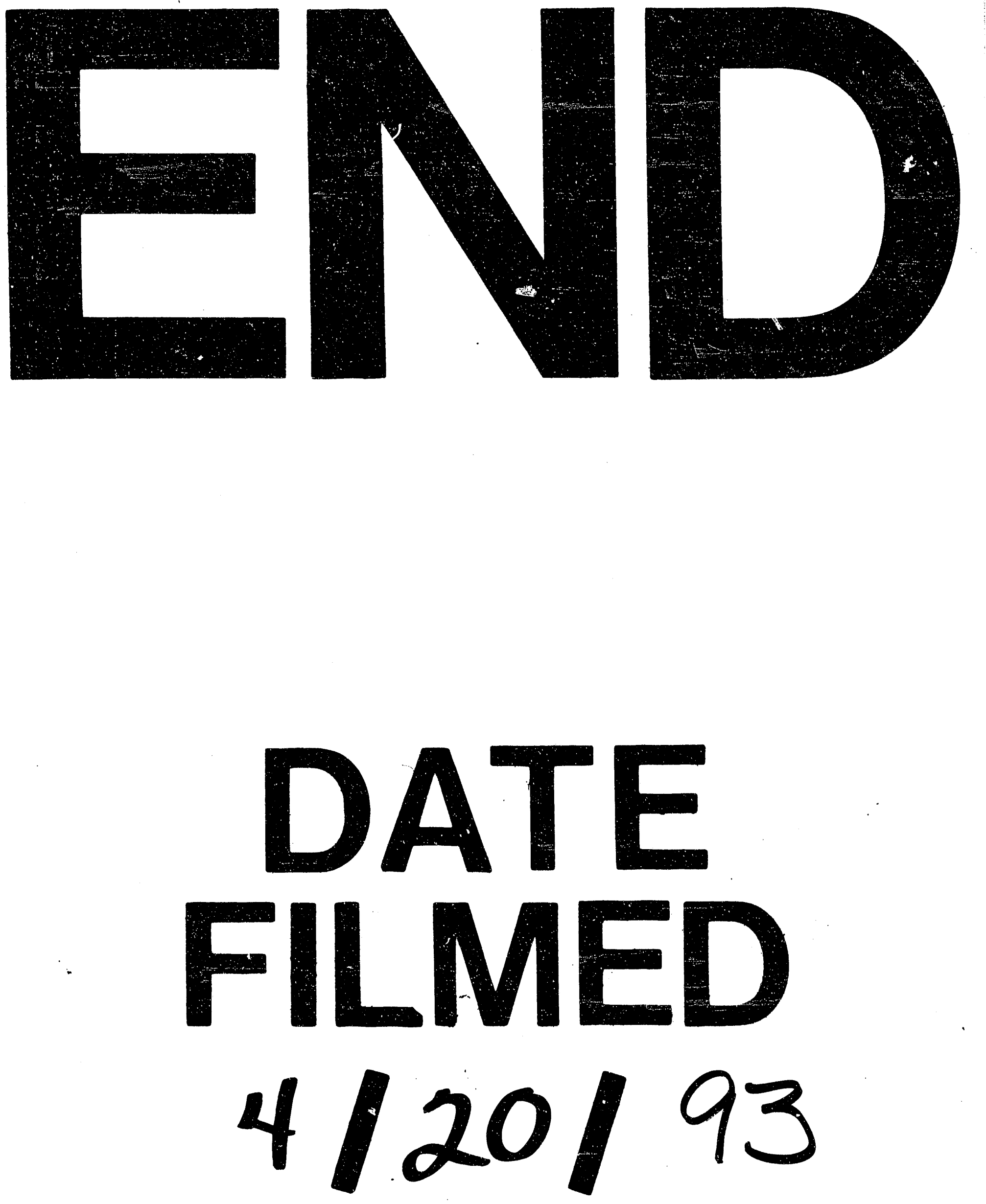

1 